\title{
FREQUENCY AND PRESENTATION OF PERSISTENT PAIN FOLLOWING BREAST CANCER SURGERY: AN OBSERVATIONAL STUDY IN A TERTIARY CARE HOSPITAL
}

\author{
Ali Sarfraz Siddiqui, Sana Zeeshan, Aliya Ahmed and Shaista Khan \\ Department of Anaesthesiology and Surgery, \\ Aga Khan University , Karachi, Pakistan
}

Background and aims: Persistent post-surgical pain (PPSP) is significant issue following breast cancer treatment. Persistent pain after mastectomy ranges from 25-60\%. Primary aim of this study was to determine the frequency of persistent pain in patients after breast cancer surgery while the secondary aim was to observe the distribution and characteristics of pain in these patients.

Methods: This prospective observational single cohort study was conducted in the Department of Anaesthesiology and the Breast Surgery Clinic of the Department of Surgery, Aga Khan University. Adult female patients with biopsy proven carcinoma of breast who were scheduled for elective definitive breast cancer surgery were enrolled in this study. Every patient was interviewed in breast clinic on follow up visits and telephonic communication with patients was also done for any missing information. Variables were defined and a form was designed for data collection. Total 120 patients were followed for up to three months after surgery and those who had persistent pain were followed up for six months after operation.

Results: Out of the 120 patients, twenty six (21.7\%) patients developed persistent post-surgical pain for up to three months while in seventeen (14.2\%) patients pain continued for up to six months after operation. Eleven patients developed pain at more than one site. Pain was distributed in axilla, chest wall, upper arm and surgical scar area. Fifteen out of twenty six patients $(57.7 \%)$, had pain in axilla.

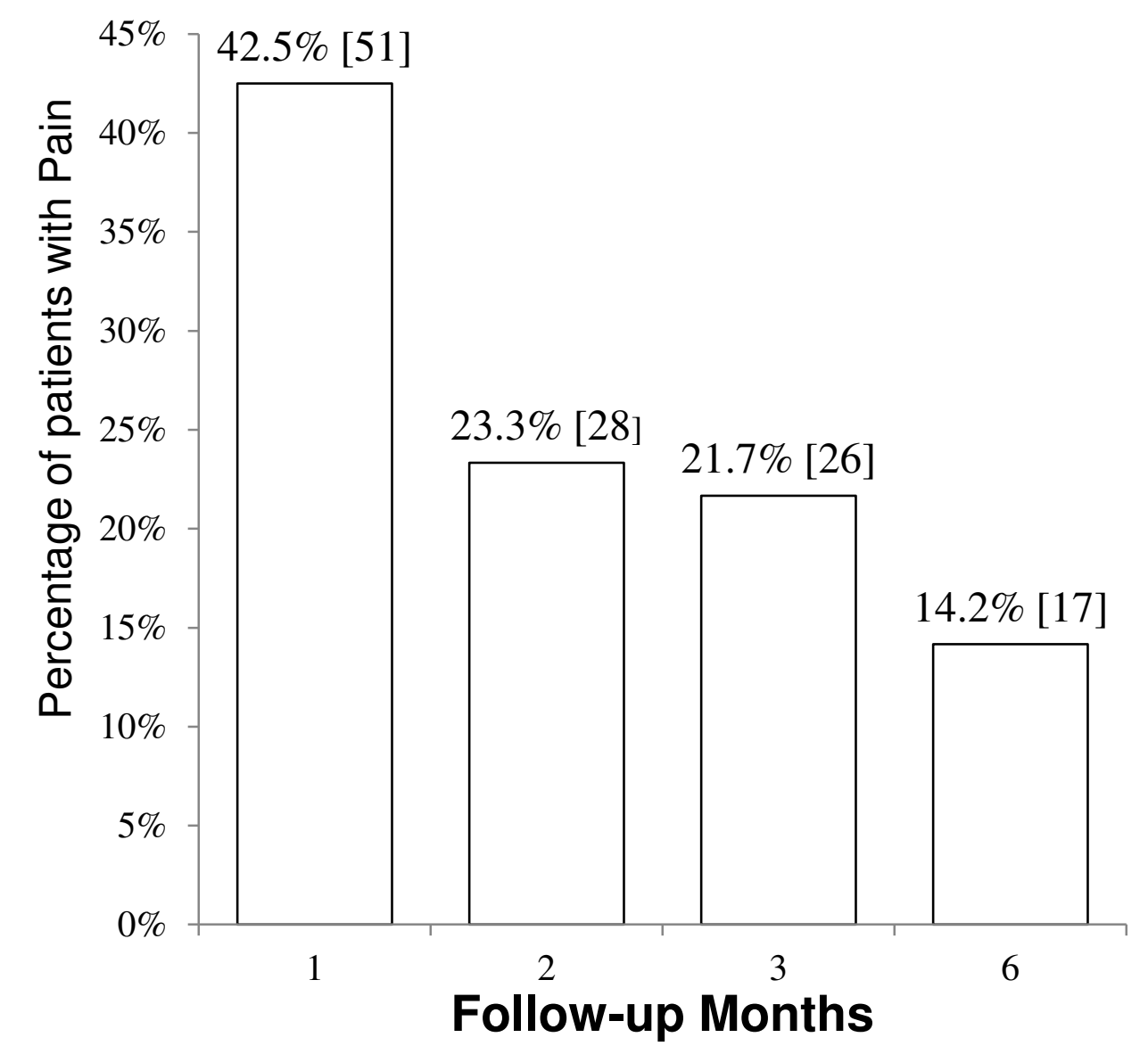

Figure 1: Patients having persistent pain with respect to follow-up $(n=120)$
Upon multiple logistic regression analysis three statistically significant risk factors of PPSP were identified at three months like younger age group of less than 50 years (OR 5.60 and pvalue 0.028), mastectomy (OR 3.97 and $p$-value 0.035) and axillary clearance (OR 2.68 and p-value 0.04).

Among those twenty six patients with PPSP, eleven (42.3\%) had burning pain, ten (38.5\%) had throbbing pain, three (11.5\%) patients had numbness while two patients had mixed character of pain. Out of the twenty six patients with persistent pain at three months, twenty one patients had mild pain, three had moderate pain while two patients had severe pain. Out of the 120 patients, five (4.16\%) patients had phantom breast pain. Pain was distributed in axilla, chest wall, upper arm and surgical scar area. Fifteen out of twenty six (57.7\%) had pain in axilla. Eighty (66.7\%) patients required radiotherapy postoperatively. Out of these eighty patients, seventeen patients developed chronic pain. Twenty one (80.8\%) patients developed mild pain, three patients developed moderate pain and two developed severe pain.

Table 1: Demographic characteristics and comorbid conditions $(n=120)$

\begin{tabular}{|c|c|}
\hline Variables & Point Estimates \\
\hline Age (years) & $50.08 \pm 12.86$ \\
\hline Weight $(\mathrm{kg})$ & $68.58 \pm 13.77$ \\
\hline Height $(\mathrm{cm})$ & $155.94 \pm 6.00$ \\
\hline BMI $\left(\mathrm{kg} / \mathrm{m}^{2}\right)$ & $28.16 \pm 5.25$ \\
\hline \multicolumn{2}{|c|}{ Comorbid of Patient } \\
\hline Diabetes Mellitus & $23(19.2 \%)$ \\
\hline Hypertension & $51(42.5 \%)$ \\
\hline Hypothyroidism & 04 (3.3\%) \\
\hline Others & $12(10 \%)$ \\
\hline
\end{tabular}

Conclusion: Persistent pain following breast cancer surgeries was found to be $21.7 \%$ in this study. Young age, mastectomy and axillary clearance are identified as significant risk factors in this study. Among those who developed persistent pain, eleven had burning pain, ten had throbbing pain, and three patients had numbness while two patients had mixed character of pain.

Dr. Ali Sarfraz Siddiqui (4257-Ane-ERC-16) Email: Sarfraz.siddiqui@aku.edu 